\title{
Robotic ureteroplasty with appendiceal onlay flap: an update on the outcomes of 18-month follow-up
}

\author{
Jie Wang ${ }^{1 \#}$, Zhihua Li ${ }^{1 \#}$, Shubo Fan ${ }^{1 \#}$, Shengwei Xiong ${ }^{1}$, Changwei Yuan ${ }^{1}$, Chang Meng ${ }^{1}$, Jun Zhang ${ }^{1}$, \\ Xiaowei Zhang ${ }^{1}$, Peng Zhang ${ }^{2}$, Mingfei Ji ${ }^{3}$, Jie Chen ${ }^{3}$, Kunlin Yang ${ }^{1}$, Xuesong Li $^{1}$ \\ ${ }^{1}$ Department of Urology, Peking University First Hospital, Institute of Urology, Peking University, National Urological Cancer Center, Beijing, \\ China; ${ }^{2}$ Department of Urology, Emergency General Hospital, Beijing, China; ${ }^{3}$ Department of Urology, Changzheng Hospital, Naval Military \\ Medical University, Shanghai, China \\ Contributions: (I) Conception and design: J Wang, S Fan; (II) Administrative support: X Li, L Zhou; (III) Provision of study materials or patients: K \\ Yang, X Li; (IV) Collection and assembly of data: Z Li, C Meng, J Zhang, X Zhang, M Ji, J Chen; (V) Data analysis and interpretation: S Xiong, C \\ Yuan, P Zhang; (VI) Manuscript writing: All authors; (VII) Final approval of manuscript: All authors. \\ \#These authors contributed equally to this work. \\ Correspondence to: Xuesong Li; Kunlin Yang. Department of Urology, Peking University First Hospital, Institute of Urology, Peking \\ University, National Urological Cancer Center, No. 8 Xishiku St., Xicheng District, Beijing 100034, China. Email: pineneedle@sina.com; \\ yangkunlin12345@163.com.
}

Background: To describe our technical experience of robotic appendiceal onlay flap ureteroplasty (RAUP) for complex ureteral stricture disease and report the updated analysis of 18-month follow-up outcomes.

Methods: Since May 2019, nine patients with right ureteral strictures have undergone RAUP in our medical centre. Patients' perioperative data and follow-up information were collected prospectively. Patients were excluded in present study if the postoperative follow-up time was less than 6 months.

Results: Eight patients were recruited. Proximal ureteric strictures were present in 5 patients, and 3 patients had middle ureteric strictures. The mean stricture length was $4.3 \mathrm{~cm}$ (range, 3.0-6.0 cm). Nephrostomy was performed in 4 patients, and 4 patients had indwelling double-J ureteral stents before they were admitted to our hospital. All operations were implemented successfully without intraoperative complications. The mean operation time was 162 minutes (range, 135-211 minutes), and the mean estimated blood loss was $78 \mathrm{~mL}$ (range, 30-200 mL). The mean postoperative hospital stay was 8 days (range, 4-12 days). No patients had high-grade postoperative complications (Clavien-Dindo III and IV) 30 days after surgery. At a mean follow-up of 18 months (range, 6-28 months), all patients were not needed further surgical intervention and could be considered successful. But 2 cases still have stable mild hydronephrosis without symptoms such as flank pain or fever.

Conclusions: RAUP is a workable option for managing long-segment $(3-6 \mathrm{~cm})$ proximal and middle ureteral strictures of the right side. The outcomes of 18-month follow-up are satisfactory.

Keywords: Ureteral stricture; appendix; onlay technique; reconstruction; robotics

Submitted Sep 24, 2021. Accepted for publication Dec 07, 2021.

doi: $10.21037 /$ tau-21-840

View this article at: https://dx.doi.org/10.21037/tau-21-840

\section{Introduction}

Clinically, various management options can be applied to address benign ureteral strictures, depending on the length and site of the ureteral stricture segment. Endoscopic therapy, such as balloon dilation and endoureterotomy, may be useful for short strictures, but endoscopic therapy was usually associated with an uninspiring success rate $(1,2)$. Undoubtedly, long-segment distal strictures of the ureter can be managed with ureteral reimplantation combined 
with a psoas hitch and/or Boari flap (3-5), and pyeloplasty can be used to handle obstruction of the ureteropelvic junction (UPJO) with a high success rate (6). However, in some cases, autologous onlay/graft technique is necessary, such as extended strictures of the proximal or middle ureter, and ureteroureterostomy with a failed outcome. Traditional management involves ileal ureter replacement or autologous kidney transplantation with a high risk of vascular or small bowel complications $(7,8)$. Urologists have committed to improving the repair effect and perform minimally invasive procedures for long-segment ureteral strictures. Several innovative methods, such as oral mucosal grafts and appendiceal onlay flaps, have been utilized to repair ureteral stricture, and encouraging outcomes have been reported by some surgeons (9-16). For ureteral strictures of appropriate length, the possibility of patients requiring ileal ureter replacement can be reduced to a certain extent by appendiceal onlay flap ureteroplasty. In general, ureteroplasty with an appendiceal onlay flap is still a rare surgical intervention, especially on robotic platforms, with few reported cases (17). However, this unique procedure is usually an option for complex ureteral strictures located in the right proximal and middle ureters. We now describe our technical experience of robotic appendiceal onlay ureteroplasty for complex ureteral stricture disease and report the updated analysis of 18 -month outcomes.

We present the following article in accordance with the STROBE reporting checklist (available at https://tau. amegroups.com/article/view/10.21037/tau-21-840/rc).

\section{Methods}

\section{Patient selection}

After obtaining approval from the Ethics Committee of Peking University First Hospital (ethical approval number: 2019SR134), we registered a cohort study in the Chinese Clinical Trial Registry (registration number: ChiCTR2000033884). We established a prospective cohort of patients with right long-segment ureteral strictures who underwent robotic appendiceal onlay flap ureteroplasty (RAUP). The clinical data of patients were collected prospectively: demographic characteristics, presenting symptoms, preoperative imaging, ureteral stricture aetiology, site, and lengths, previous management, operative details and postoperative complications (according to the Clavien-Dindo classification), postoperative hospital stay and follow-up data. The outcomes of patients were evaluated through two aspects, including their postoperative symptoms and imaging examinations, such as upper urinary tract imaging urodynamics examination (IUE) which has been described in our previous studies $(18,19)$, functional cine magnetic resonance urography (cine MRU), and computed tomography urography (CTU). According to our experience, the following conditions are considered to be complex ureteral stricture diseases. Endoscopic ureteral treatment or repair surgery have failed. Severe scar adhesion in the stricture segment makes end-to-end anastomosis impossible. The double-J stent must be indwelled for a long time and cannot be removed.

\section{Surgical techniques}

Surgery was performed under general anaesthesia with nasotracheal intubation using da Vinci Si surgical systems (Intuitive Surgical, Sunnyvale, CA, USA). The patients were placed in a lateral decubitus position with the right side elevated by $90^{\circ}$. The port placement included one camera trocar, three robot arm trocars and two assistant trocars, as shown in Figure 1A,1B.

Once the robot was docked at $90^{\circ}$ to the patient and the $30^{\circ}$ camera was equipped, an incision was made along the right white Toldt line, and then the colon was mobilized and reflected medially. At the same time, the appendix was inspected for adequate length and a generally healthy appearance. After the retroperitoneum was entered, the renal pelvis and ureter were identified by sharp and blunt dissection. From our experience, ureteral stenoses can be identified via the following techniques. The site and length of ureteral strictures were estimated according to preoperative anterograde and retrograde urography and three-dimensional reconstruction of computed tomography (3D-CT), which can also be used for intraoperative realtime navigation. For patients with nephrostomy, the nephrostomy was filled with saline so that the upper ureter became distended. Indocyanine green (ICG) can be injected intravascularly or intraureterally and combined with nearinfrared fluorescence imaging to observe the ureteral stenosis segment.

Once the length of the ureteral stricture was revealed and deemed to be too long for end-to-end anastomosis, the narrow segment was incised longitudinally along the anterior wall until healthy pink ureteric mucosa was seen on both sides of the stricture (Figure 1C,1D). If the ureteral lumen was obliterative, we resected the ureteral obliterative segment and then anastomosed the normal ureteral tissue with the posterior wall to create a new ureteral plate 

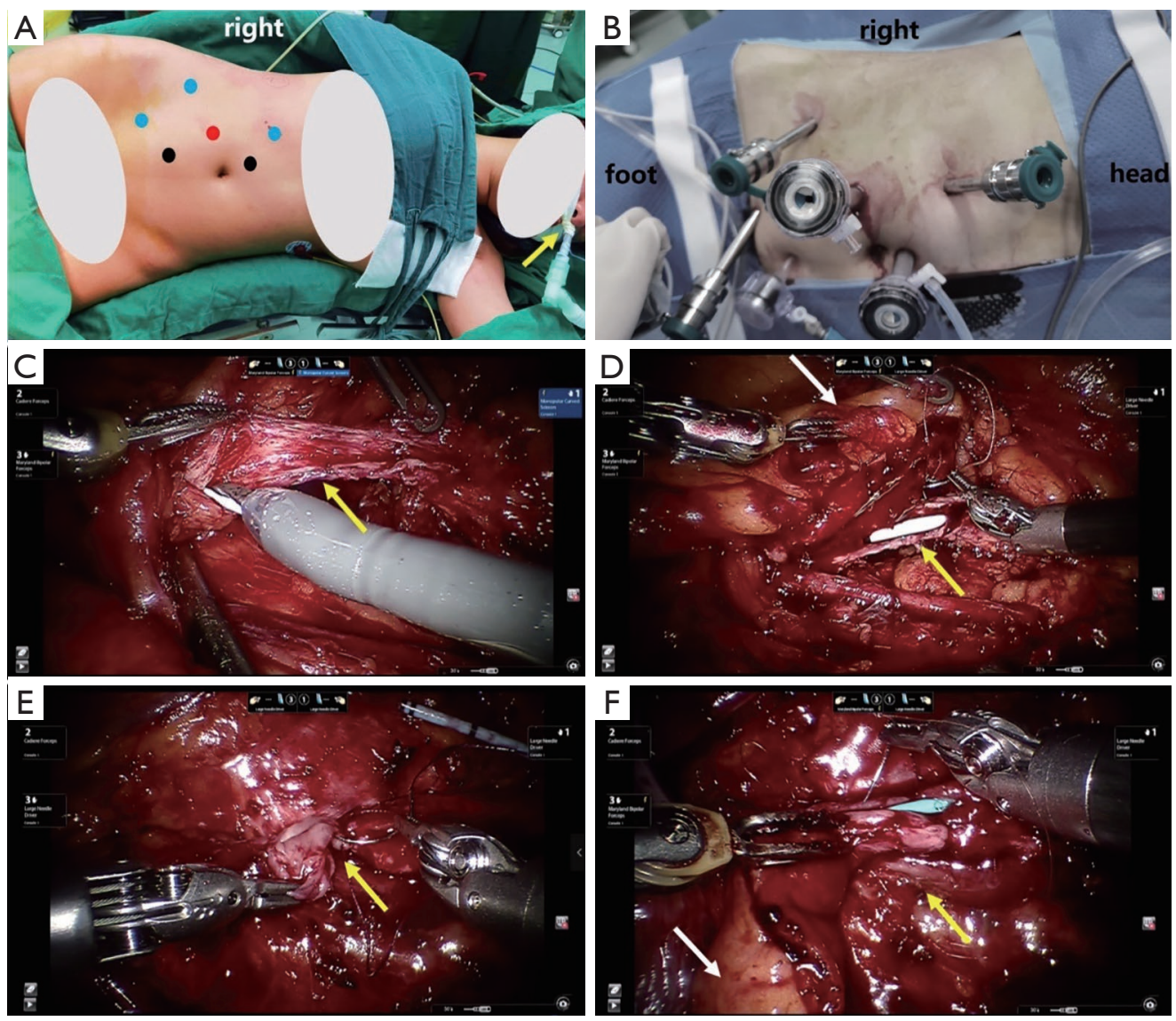

Figure 1 Patient position \& port placement, and the examples of different degrees of ureteral strictures. (A) Patients were placed in a lateral decubitus position with the right side elevated by $90^{\circ}$. Laparoscopic trocar was marked as a red dot. Robotic arm trocars were marked as blue dots. Assistant trocars were marked as black dots. General anaesthesia with nasotracheal intubation (yellow arrow); (B) port placement for robotic appendiceal onlay flap ureteroplasty; (C) the posterior wall of the ureteral stricture was smooth and flat (yellow arrow); (D) the appendiceal onlay flap with mesentery was anastomosed on the anterior wall (white arrow) to cover the posterior wall (yellow arrow); (E) the lumen of the ureteral stricture was obliterative, the obliterative segment was resected, and normal ureteral tissue of the posterior wall was anastomosed. Then, a new ureteral plate was created (yellow arrow); (F) the appendiceal onlay flap with mesentery was anastomosed on the anterior wall (white arrow) to cover the posterior wall (yellow arrow).

(Figure $1 E, 1 F$ ). The length of defect of the ureteral anterior wall was accurately measured using a ureteric catheter. After the root of the appendix was clamped by Hem-olok, the appendix was separated from the caecum with its mesoappendix preserved. The length was equal to the ureteral defect and detubularized along its antimesenteric border. A 6-F double-J ureteral stent was placed over a wire within the ureter before anastomosis of the ureteral defect and appendiceal onlay flap (Figure $1 D, 1 F$ ). The caecal end of the appendiceal onlay flap was anastomosed to the distal ureter, and the opposite end was anastomosed to the proximal ureter. A 5-0 Monocryl suture (Ethicon, USA) was used for continuous anastomoses (Figure 2). Finally, the omentum was wrapped around the onlay flap and ureter, and a drain was placed near the anastomosis.

\section{Postoperative management and follow-up}

Generally, the patient was given injectable antibiotics for five days. The drain was removed once the output was less than $50 \mathrm{~mL}$ (4-6 days) after the operation. The Foley catheters were usually removed after one week. Kidneyureter-bladder (KUB) was performed routinely before patient discharge to confirm that the double-J stent was in 

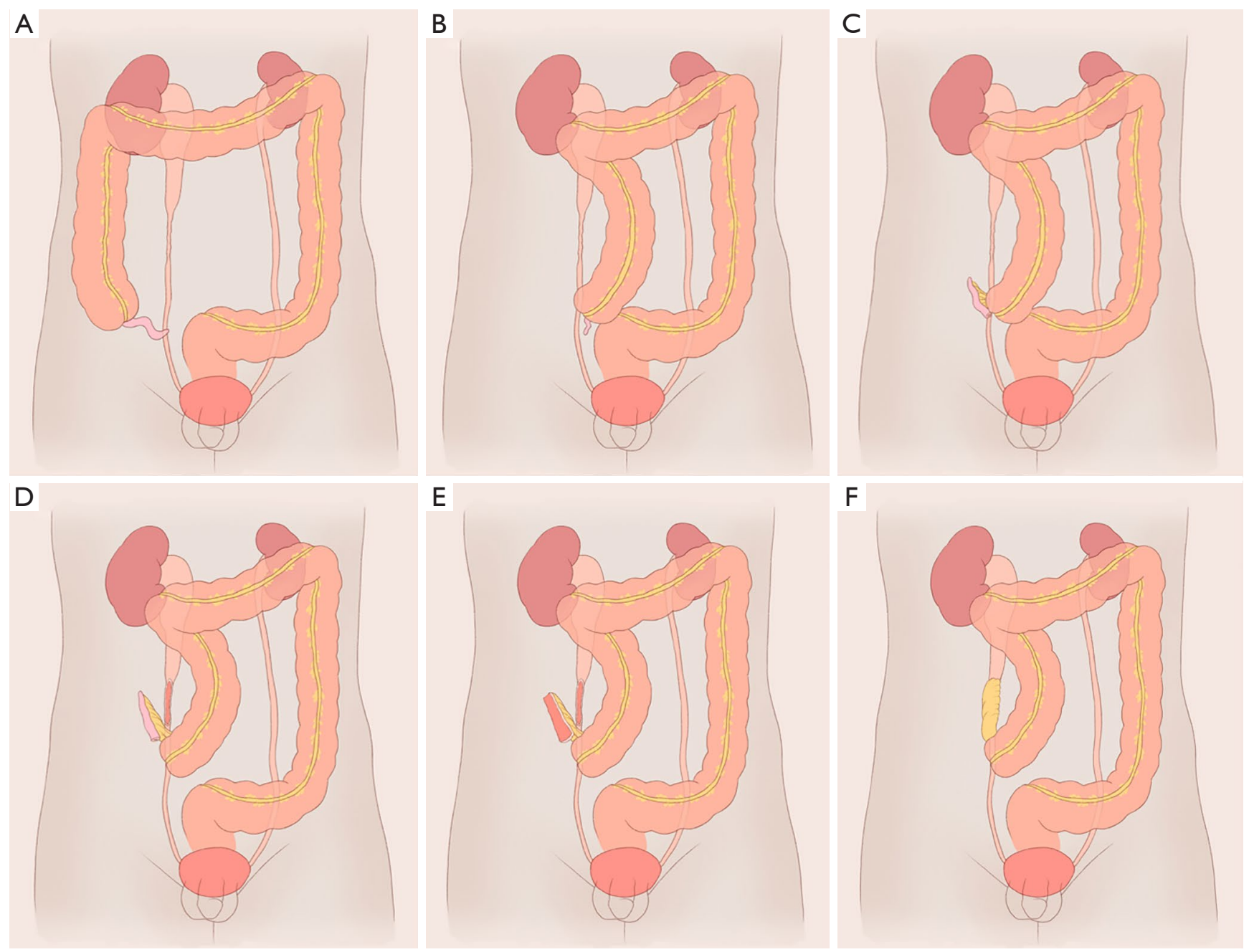

Figure 2 Schematic diagram of appendiceal onlay flap ureteroplasty. (A) Exposing the area of operation and probing the appendix; (B) mobilizing the right colon; (C) resecting the appendix from the caecum with the mesoappendix preserved; (D) managing the ureteral strictures, rebuilding the posterior wall, and leaving a defect in the anterior wall; (E) creating an appendiceal onlay flap with its mesoappendix preserved, and its length was the same as the defect of the anterior wall; (F) the appendiceal onlay flap was anastomosed on the anterior wall.

the proper position. For patients with nephrostomy, the tube was clamped but not removed two weeks after surgery. The preoperative urography of case 7 was shown in Figure $3 \mathrm{~A}$. And this patient with nephrostomy underwent IUE after removing double-J stents (Figure 3B), which has been described previously (19). The preoperative urography of case 4 was shown in Figure $3 C$. And this patient without nephrostomy underwent functional cine MRU after removing the double-J stents (20) (Figure 3D). According to our experience, ureteroscopy was not recommended for postoperative patients. Then, patients underwent an ultrasound examination three months after surgery. Finally, patients paid attention to their symptoms, such as flank pain or fever, and repeat ultrasound or CT was performed six months later and then annually. The patient outcome was evaluated by two criteria. Subjective success was defined as the resolution of flank pain without a double-J stent and nephrostomy. Objective success was defined as the absence of ureteral obstruction on imaging examinations.

\section{Statistical analysis}

Data were analyzed using SPSS software version 22.0 (IBM Corporation, Armonk, NY, USA). Measurement 

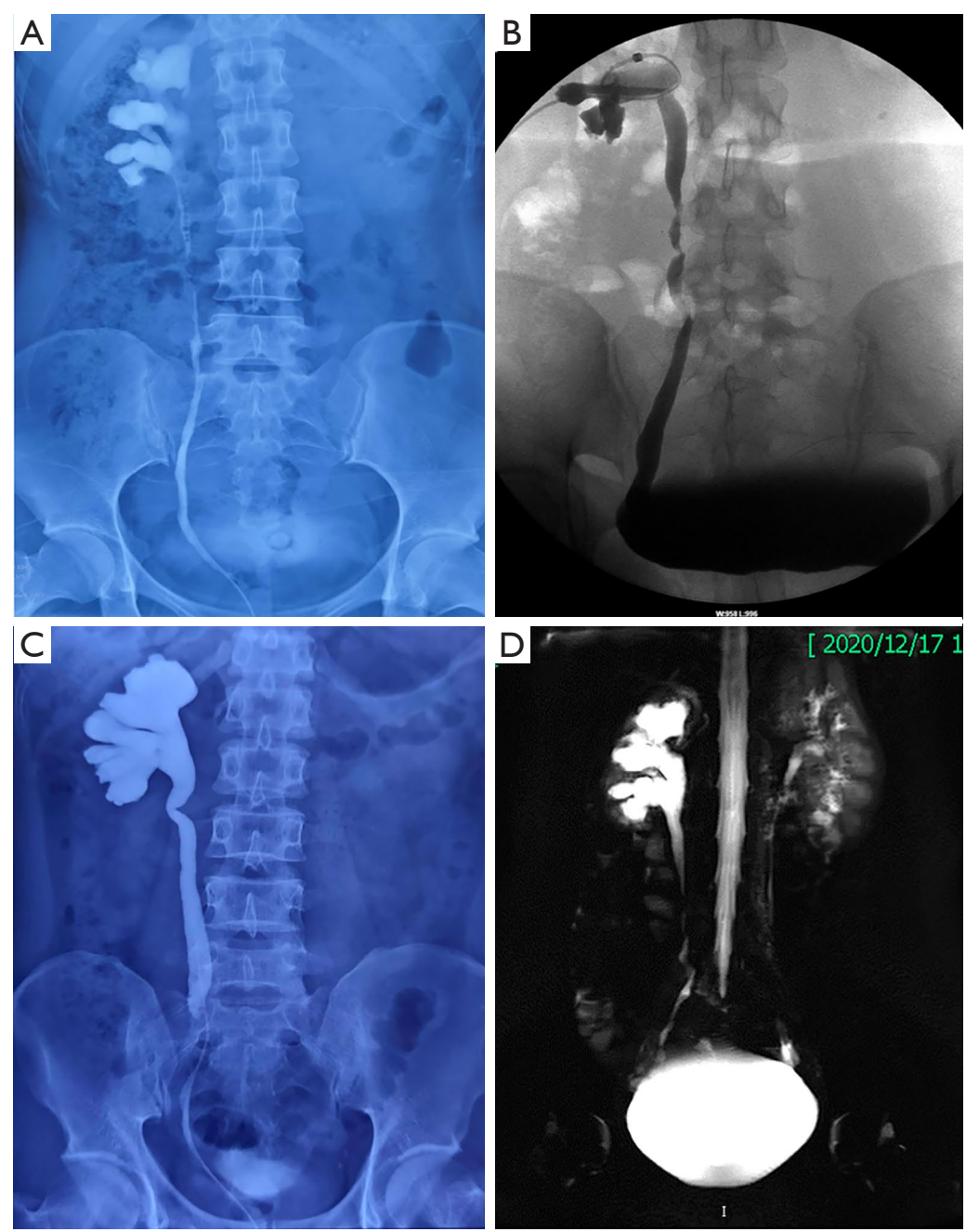

Figure 3 Imaging examinations of case 4 and case 7. (A) Preoperative anterograde and retrograde urography of case 7; (B) the postoperative IUE of case 7 showed that the ureter was unobstructed; (C) preoperative anterograde and retrograde urography of case 4; (D) the postoperative cine MRU of case 4 showed that the ureter was unobstructed. IUE, imaging urodynamics examination; MRU, magnetic resonance urography.

data are expressed as the mean (range), and enumeration data are expressed as numbers (percentages). The variables mentioned in the manuscript were all in accordance with the normal distribution.

\section{Ethical statement}

The study was conducted in accordance with the Declaration of Helsinki (as revised in 2013). The study was approved by Ethics Committee of Peking University First Hospital (No. 2019SR134) and informed consent was taken from all the patients.

\section{Results}

Since May 2019, nine patients with right ureteral strictures have undergone RAUP in our medical centre. One patient was excluded in present study because his postoperative follow-up period was less than 6 months. The demographic and characteristics are summarized in Table 1. Eight patients were between 28 and 46 years old (mean age 38.5), including 5 men and 3 women, and the mean body mass index (BMI) was $24.3 \mathrm{~kg} / \mathrm{m}^{2}$ (range, $22.0-31.5 \mathrm{~kg} / \mathrm{m}^{2}$ ). All patients were given an American Society of Anesthesiologists score (ASA) of II. Of the ureteral strictures, six patients had iatrogenic injuries with urolithiasis treatment, two patients were 
Table 1 Demographic and preoperative characteristics

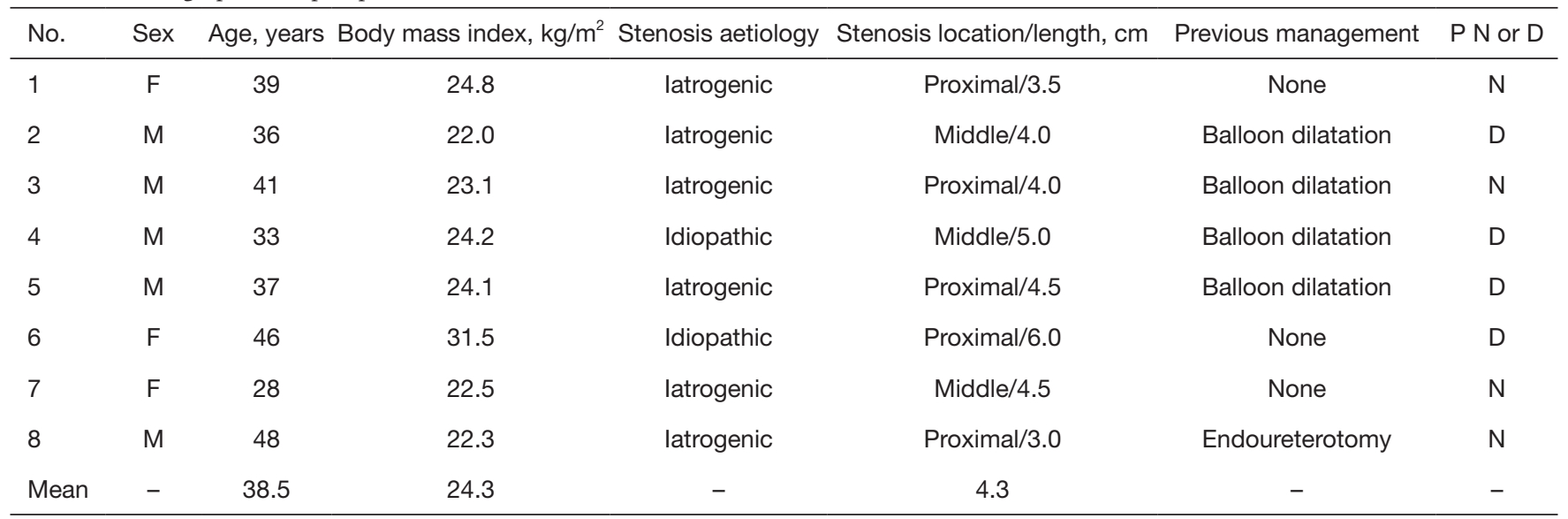

$\mathrm{P} \mathrm{N}$ or $\mathrm{D}$, preoperative nephrostomy or double-J stents.

idiopathic in origin, and the causes were unclear. The sites of strictures were the proximal ureter in 5 patients and the mid-ureter in 3 patients. The mean stricture length, as measured during the operations, was $4.3 \mathrm{~cm}$ (range, 3.0$6.0 \mathrm{~cm}$ ). All patients had right flank pain and hydronephrosis present on ultrasound preoperatively. Nephrostomy was performed in 4 patients and the other 4 patients had indwelling double-J ureteral stents before they came to our hospital. The right renal functions were significantly lower than the contralateral side, but both were greater than $20 \%$ of total renal function.

All operations were performed successfully without intraoperative complications.

The preoperative and postoperative data of all patients are summarized in Table 2. The mean operative time was 162 minutes (range, 135-211 minutes), and the mean estimated blood loss was $78 \mathrm{~mL}$ (range, 30-200 mL). None of the patients needed a blood transfusion. The mean length of postoperative hospital stay was 8 days (range, 4-12 days). No high-grade postoperative complications (Clavien-Dindo III and IV) occurred within 30 days of surgery. One male patient developed fever during hospitalization after surgery, and responded well to injectable antibiotics. One female patient found that her double-J ureteral stent protruded out of the body two months after the operation, and she experienced urethral pain when she urinated. She started oral antibiotics, and her cine MRU 3 months after the operation showed that the right renal calyces and pelvis, and right proximal and middle ureter were dilated; regular peristalsis could be seen in the right renal pelvis and ureter. Then, ultrasound was performed, which showed that the right renal pelvis and ureter were slightly dilated, and a hypoechoic mass with no blood flow in the right ureteral orifice, considering that it was an appendiceal mucus plug, was observed continuously. Six months after the operation, the right back pain of this patient disappeared completely. Ultrasound and CT showed that the right hydronephrosis was significantly relieved before the operation (Figure 4). We believe that patients need time to recover after surgery, and individual differences are obvious. The mean followup duration was 18 months (range, 6-28 months), and all patients' double-J ureteral stents and nephrostomy tubes were removed two or three months after the operations. The upper urinary tracts of all patients were unobstructed, as shown by imaging examinations which meant that the objective success rate was $100 \%$. But 2 cases still have stable mild hydronephrosis without symptoms such as flank pain or fever. Symptoms such as flank pain and fever disappeared in all patients, and no further surgical intervention were required. The subjective success rate was considered to be $100 \%$.

\section{Discussion}

Ureteral replacement is an important procedure for addressing complex ureteral strictures in the field of ureteral reconstruction. At the present time, autologous tissues can be used to repair ureteral strictures, including the intestines (ileum and colon), oral mucosa (lingual mucosa and buccal mucosa), and appendix (11,13,21-23). Melnikoff first attempted to use the appendix to replace the ureter in 1912 (15). Gradually, some surgeons have 
Table 2 Intraoperative and postoperative data

\begin{tabular}{|c|c|c|c|c|c|c|}
\hline No. & $\begin{array}{l}\text { Operation } \\
\text { time, } \min \end{array}$ & $\begin{array}{c}\text { Estimated } \\
\text { blood loss, } \mathrm{mL}\end{array}$ & $\begin{array}{l}\text { Posterior augmented } \\
\text { anastomosis, yes/no }\end{array}$ & $\begin{array}{c}\text { Postoperative } \\
\text { hospital stays, days }\end{array}$ & $\begin{array}{l}\text { Follow-up, } \\
\text { months }\end{array}$ & $\begin{array}{l}\text { Postoperative } \\
\text { complications }\end{array}$ \\
\hline 1 & 135 & 50 & No & 9 & 28 & None \\
\hline 3 & 211 & 50 & Yes & 8 & 25 & Fever \\
\hline 4 & 182 & 200 & No & 12 & 24 & None \\
\hline 6 & 137 & 30 & No & 6 & 12 & D-J stent prolapse \\
\hline 7 & 139 & 100 & Yes & 4 & 12 & None \\
\hline 8 & 163 & 60 & Yes & 5 & 6 & None \\
\hline
\end{tabular}
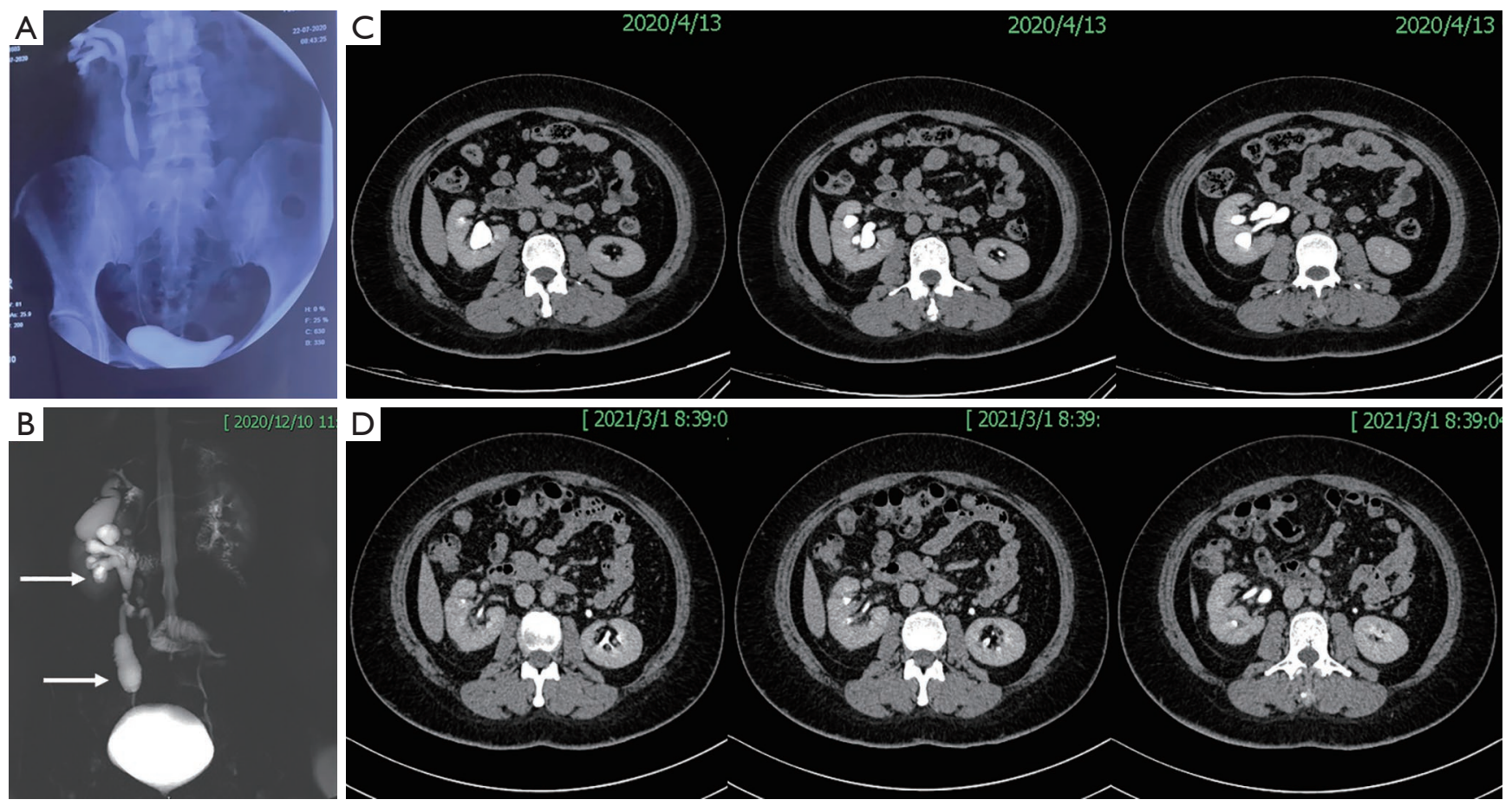

Figure 4 Imaging examinations of case 6. (A) Preoperative retrograde urography of case 6, which had the longest ureteral strictures (6 cm) in our cohort; (B) the cine MRU at 3 months after the operation showed that the right renal calyces, pelvis, and the proximal and middle ureters were dilated (white arrows); (C) the preoperative enhanced CT showed right hydronephrosis; (D) enhanced CT 6 months after surgery showed that right hydronephrosis was significantly relieved. MRU, magnetic resonance urography; CT, computed tomography.

reported a few cases of this procedure, appendiceal interposition (end-to-end anastomosis), mainly for ureteral strictures in paediatric patients (19). The concept of onlay grafts, which were first used to manage urethral stricture disease, was first applied to ureteral reconstruction in 2009.
Reggio et al. described laparoscopic appendiceal onlay flap ureteroplasty, which was successful with 8 months of followup (14). However, there were no more than several cases of either the appendiceal interposition or appendiceal onlay technique. Duty et al. reported on a series of six patients 
in which all patients achieved objective success (defined as absence of obstruction on imaging) after appendiceal onlay ureteroplasty. They believed that the method of appendiceal interposition carries a significant risk of stricture recurrence compared with the appendiceal onlay flap technique (15). However, Yarlagadda et al. first tried to perform roboticassisted laparoscopic appendiceal interposition for a $5 \mathrm{~cm}$ obliterative right ureteral stricture and achieved success (24). In the world literature, the longest ureteral stenoses repaired with an appendiceal onlay flap and the appendiceal interposition were 6 and $12 \mathrm{~cm}$, respectively $(15,16)$. From this perspective, end-to-end anastomosis with a section of appendix can repair longer ureteral defects in contrast with the appendiceal onlay technique. In addition, Burns et al. reported eleven adult patients who underwent ureteral appendiceal interposition for right or left ureteral strictures and achieved an $83.3 \%$ success rate (25). Objectively speaking, we only perform appendiceal onlay flap ureteroplasty at present but do not deny the feasibility of appendiceal interposition.

Robotic assistance for ureteral reconstruction has incomparable advantages: magnified view, three-dimensional visualization, and articulated instruments, which facilitate delicate suturing. To our knowledge, we report herein the largest cohort of patients undergoing appendiceal onlay flap ureteroplasty with da Vinci Si surgical systems in a single centre. We have published an appendiceal onlay ureteroplasty case series with four robotic procedures previously (19). The present manuscript provided a mean 18-month follow-up on previous patients and experience with four additional cases. Appendix was used as an onlay flap to repair ureteral strictures and has several advantages. The wall of the appendix is similar to that of the ureter. The appendiceal mucosa in contact with urine is not large, resulting in minimal urine absorption and avoidance of electrolyte imbalances in patients compared with patients who underwent ileal ureter replacement. The appendix also has peristalsis directed to its base and allows urine to pass smoothly. The blood supply of the appendiceal onlay flap is better than that of the oral mucosa graft because the appendix mesoappendix is preserved completely. However, its disadvantages are as follows. The length of the appendix is limited, so the length of ureteral stricture that can be repaired are limited. Given the limitations of anatomical proximity, and the mobility of the appendix which allowed by mesoappendix, the appendiceal onlay is only suitable for repairing the right ureteral stricture, especially in adults. However, the oral mucosa can be used on both sides. Deyl et al. reported the first case of total left ureteral reconstruction with appendix interposition for a 3-monthold child (26). Komyakov et al. reported their experience with four successful cases with appendix interposition repair of left pelvic ureteral stricture in adults (16). Based on our experience, an appendiceal onlay flap is mainly applicable to repair right proximal and middle ureter long-segment strictures. However, Jun et al. believed that appendiceal ureteral reconstruction for distal ureteral stricture was suitable for patients already with a small bladder capacity or patients who have undergone pelvic radiation, which cannot be treated with a Boari flap (17). Asghar et al. reported that they had success with an appendiceal bypass (interposition) procedure to repair radiation-induced distal ureteral strictures (27).

In our institution, for complex strictures of the right proximal and middle ureters, we tend to perform RAUP. However, nasotracheal intubation and oral cavity disinfection were prepared in advance for lingual mucosa graft ureteroplasty because the conditions of the appendix may not be suitable for repairing the ureter due to, for example, inadequate length or surrounding fibrosis secondary to previous inflammation. Moreover, for long-segment or multi-segment ureteral strictures caused by pelvic radiotherapy, which cannot be treated with appendiceal or oral mucosal onlay ureteroplasty, to improve the success rate of ureteral reconstruction, we tend to perform ileal ureter replacement for patients. Of course, bilateral long-segment ureteral strictures can only be reconstructed with ileal ureter replacement (28). We believe that an individualized management strategy must be developed for each patient with ureteral stricture. For patients with 3-6 cm right proximal and middle ureteral stenoses that were not eligible for direct anastomosis, RAUP could be an effective option and ought to be familiar to reconstructive urologists.

The major limitations of this study were the small sample size and lack of comparison with other surgical methods, although the data on the patients were collected prospectively. A larger patient cohort, longer follow-up period and comparative studies are needed. In addition, this study is a report of single center experience with RAUP. In order to formulate more reasonable ureteral repair strategies, multi-institutional studies should be conducted in future studies.

\section{Conclusions}

RAUP is a workable option for managing long-segment 
(3-6 cm) proximal and middle ureteral strictures of the right side. The outcomes of 18-month follow-up are satisfactory.

\section{Acknowledgments}

We acknowledge the excellent work of the medical illustrator Madam Xinying Ma, who drew figures for us. Funding: None.

\section{Footnote}

Reporting Checklist: The authors have completed the STROBE reporting checklist. Available at https://tau. amegroups.com/article/view/10.21037/tau-21-840/rc

Data Sharing Statement: Available at https://tau.amegroups. com/article/view/10.21037/tau-21-840/dss

Peer Review File: Available at https://tau.amegroups.com/ article/view/10.21037/tau-21-840/prf

Conflicts of Interest: All authors have completed the ICMJE uniform disclosure form (available at https://tau.amegroups. com/article/view/10.21037/tau-21-840/coif). XL serves as an unpaid editorial board member of Translational Andrology and Urology. The other authors have no conflicts of interest to declare.

Ethical Statement: The authors are accountable for all aspects of the work in ensuring that questions related to the accuracy or integrity of any part of the work are appropriately investigated and resolved. The study was conducted in accordance with the Declaration of Helsinki (as revised in 2013). The study was approved by Ethics Committee of Peking University First Hospital (No. 2019SR134) and informed consent was taken from all the patients.

Open Access Statement: This is an Open Access article distributed in accordance with the Creative Commons Attribution-NonCommercial-NoDerivs 4.0 International License (CC BY-NC-ND 4.0), which permits the noncommercial replication and distribution of the article with the strict proviso that no changes or edits are made and the original work is properly cited (including links to both the formal publication through the relevant DOI and the license). See: https://creativecommons.org/licenses/by-nc-nd/4.0/.

\section{References}

1. Lucas JW, Ghiraldi E, Ellis J, et al. Endoscopic Management of Ureteral Strictures: an Update. Curr Urol Rep 2018;19:24.

2. Hafez KS, Wolf JS Jr. Update on minimally invasive management of ureteral strictures. J Endourol 2003;17:453-64.

3. Wenske S, Olsson CA, Benson MC. Outcomes of distal ureteral reconstruction through reimplantation with psoas hitch, Boari flap, or ureteroneocystostomy for benign or malignant ureteral obstruction or injury. Urology 2013;82:231-6.

4. Stein R, Rubenwolf P, Ziesel C, et al. Psoas hitch and Boari flap ureteroneocystostomy. BJU Int 2013;112:137-55.

5. Asghar AM, Lee RA, Yang KK, et al. Robot-assisted distal ureteral reconstruction for benign pathology: Current state. Investig Clin Urol 2020;61:S23-32.

6. Davenport K, Minervini A, Timoney AG, et al. Our experience with retroperitoneal and transperitoneal laparoscopic pyeloplasty for pelvi-ureteric junction obstruction. Eur Urol 2005;48:973-7.

7. Kocot A, Kalogirou C, Vergho D, et al. Long-term results of ileal ureteric replacement: a 25-year single-centre experience. BJU Int 2017;120:273-9.

8. Eisenberg ML, Lee KL, Zumrutbas AE, et al. Longterm outcomes and late complications of laparoscopic nephrectomy with renal autotransplantation. J Urol 2008;179:240-3.

9. Naude JH. Buccal mucosal grafts in the treatment of ureteric lesions. BJU Int 1999;83:751-4.

10. Kroepfl D, Loewen H, Klevecka V, et al. Treatment of long ureteric strictures with buccal mucosal grafts. BJU Int 2010;105:1452-5.

11. Zhao LC, Weinberg AC, Lee Z, et al. Robotic Ureteral Reconstruction Using Buccal Mucosa Grafts: A Multiinstitutional Experience. Eur Urol 2018;73:419-26.

12. Lee Z, Keehn AY, Sterling ME, et al. A Review of Buccal Mucosa Graft Ureteroplasty. Curr Urol Rep 2018;19:23.

13. Li B, Xu Y, Hai B, et al. Laparoscopic onlay lingual mucosal graft ureteroplasty for proximal ureteral stricture: initial experience and 9-month follow-up. Int Urol Nephrol 2016;48:1275-9.

14. Reggio E, Richstone L, Okeke Z, et al. Laparoscopic ureteroplasty using on-lay appendix graft. Urology 2009;73:928.e7-10.

15. Duty BD, Kreshover JE, Richstone L, et al. Review of 
appendiceal onlay flap in the management of complex ureteric strictures in six patients. BJU Int 2015;115:282-7.

16. Komyakov B, Ochelenko V, Guliev B, et al. Ureteral substitution with appendix. Int J Urol 2020;27:663-9.

17. Jun MS, Stair S, Xu A, et al. A Multi-Institutional Experience With Robotic Appendiceal Ureteroplasty. Urology 2020;145:287-91.

18. Yang Y, Li X, Xiao Y, et al. A modified Whitaker test (upper urinary tract videourodynamics) using for evaluating complex upper urinary tract reconstruction surgical effect. Transl Androl Urol 2021;10:336-44.

19. Wang J, Xiong S, Fan S, et al. Appendiceal Onlay Flap Ureteroplasty for the Treatment of Complex Ureteral Strictures: Initial Experience of Nine Patients. J Endourol 2020;34:874-81.

20. Zhu WJ, Ma MM, Zheng MM, et al. Cine magnetic resonance urography for postoperative evaluation of reconstructive urinary tract after ileal ureter substitution: initial experience. Clin Radiol 2020;75:480.e1-9.

21. Ordorica R, Wiegand LR, Webster JC, et al. Ureteral replacement and onlay repair with reconfigured intestinal segments. J Urol 2014;191:1301-6.

22. Gomez-Gomez E, Malde S, Spilotros M, et al. A tertiary experience of ileal-ureter substitution: Contemporary

Cite this article as: Wang J, Li Z, Fan S, Xiong S, Yuan C, Meng C, Zhang J, Zhang X, Zhang P, Ji M, Chen J, Yang K, Li X. Robotic ureteroplasty with appendiceal onlay flap: an update on the outcomes of 18-month follow-up. Transl Androl Urol 2022;11(1):20-29. doi: 10.21037/tau-21-840 indications and outcomes. Scand J Urol 2016;50:192-9.

23. Fan S, Yin L, Yang K, et al. Posteriorly augmented anastomotic ureteroplasty with lingual mucosal onlay grafts for long proximal ureteral strictures: 10 cases of experience. J Endourol 2021;35:192-9.

24. Yarlagadda VK, Nix JW, Benson DG, et al. Feasibility of Intracorporeal Robotic-Assisted Laparoscopic Appendiceal Interposition for Ureteral Stricture Disease: A Case Report. Urology 2017;109:201-5.

25. Burns ZR, Sawyer KN, Selph JP. Appendiceal Interposition for Ureteral Stricture Disease: Technique and Surgical Outcomes. Urology 2020;146:248-52.

26. Deyl RT, Averbeck MA, Almeida GL, et al. Appendix interposition for total left ureteral reconstruction. J Pediatr Urol 2009;5:237-9.

27. Asghar AM, Lee Z, Lee RA, et al. Robotic Ureteral Reconstruction in Patients with Radiation-Induced Ureteral Strictures: Experience from the Collaborative of Reconstructive Robotic Ureteral Surgery. J Endourol 2021;35:144-50.

28. Zhong W, Hong P, Ding G, et al. Technical considerations and outcomes for ileal ureter replacement: a retrospective study in China. BMC Surg 2019;19:9. 